\title{
BadIdeas for Usability and Design of Medicine and Healthcare Sensors
}

\author{
Paula Alexandra Silva ${ }^{1}$ and Kristof Van Laerhoven ${ }^{2}$ \\ ${ }^{1}$ Lancaster University, Computing/Infolab21 \\ Lancaster, LA1 4YR, UK \\ palexalgmail. com \\ ${ }^{2}$ Darmstadt University of Technology, Department of Computer Science \\ 64289 Darmstadt, Germany \\ kristof@mis.tu-darmstadt.de
}

\begin{abstract}
This paper describes the use of a technique to improve design and to develop new uses and improve usability of user interfaces. As a case study, we focus on the design and usability of a research prototype of an actigraph - electronic activity and sleep study device - the Porcupine. The proposed BadIdeas technique was introduced to a team of students who work with this sensor and the existing design was analysed using this technique. The study found that the BadIdeas technique has promising characteristics that might make it an ideal tool in the prototyping and design of usability-critical appliances.
\end{abstract}

Keywords: Human-Computer Interaction, Usability Engineering, Medical Informatics, Human Factors, Innovation, BadIdeas.

\section{Introduction}

Wearable biomedical sensors still have many unsolved challenges to tackle. Along with reliability, security or communication infrastructure issues, usability comes first in several studies and projects $[4,6,7]$. When searching the literature for usability studies of wearable sensors, we realise that they are scarce to inexistent; this leads us to believe that this is still a largely unexplored domain. As fields such as ubiquitous computing evolve, usability for wearable sensors will gain importance, as it may be the safest way to facilitate the entrance of sensors into our daily routines.

At present, there are many ongoing projects (e.g., [6] or [4]) exploring eHealth and sensors technology potential. This will improve not only doctors' and care takers' professional work but also, and more important, patients' and citizens' quality of life in general. Certainly these will necessarily cause an impact in sensors usability investigation, promoting the development of this domain.

\section{Background}

The BadIdeas technique claims to favour divergent and critical thinking [3]. These are two fundamental characteristics to develop new uses and applications for a given technological component (as frequently done in ubiquitous computing), or to assess and improve the quality of solutions (as demanded in the process of any technology based 
project). The BadIdeas technique asks a certain group of participants to think of bad, impractical, even silly ideas within a specific domain and then uses a series of prompts to explore the domain, while directing participants into transforming their initial wacky thoughts into a good practical idea. As detailed in [3], this technique obeys four phases: i) generation of (bad) ideas; ii) analysis: what, why and when not; iii) turning things around; and iv) making it good. The second step that elicits a series of prompts (see Table 1) is crucial, as it allows and induces us to a deep and elaborated analysis of the problem domain. Prompt questions for BadIdeas (adapted from [3])

\begin{tabular}{|l|l|}
\hline \multicolumn{1}{|c|}{ The bad } & \multicolumn{1}{|c|}{ The good } \\
\hline 1. What is bad about this idea? ....... & $\begin{array}{l}\text { 1. What is good about this idea? } \\
\text { 2. Why is this a bad thing? }\end{array}$ \\
$\begin{array}{l}\text { 2. Why is this a good thing? } \\
\text { bad? }\end{array}$ & $\begin{array}{l}\text { 3. Anything sharing this feature that is not } \\
\text { good? }\end{array}$ \\
$\begin{array}{l}\text { 4. If so what is the difference? } \\
\text { 5. Is there a different context where this is the difference? } \\
\text { would be good? }\end{array}$ & $\begin{array}{l}\text { 5. Is there a different context where this } \\
\text { would be bad? }\end{array}$ \\
\hline
\end{tabular}

By aiming at bad ideas, this technique reduces subjects' personal attachment towards their 'good ideas' and fosters 'out-of-the-box' thinking, thereby bringing out new ideas. Additionally, by stimulating critique and interrogation it largely raises a subject understanding of almost any domain.

Our ongoing study of the BadIdeas technique shows that we can use this method not only to explore a general problem, as we ought to do when thinking about new uses for a certain technological component (see 3.2.), but also to solve a particular issue of a problem, such as a usability flaw (see 3.3). Moreover, BadIdeas have the advantage of being potentially used by anybody, facilitating various types of user, such as doctors, care-givers or patients, to be involved in the development process.

The Porcupine (Fig. 1) [8] is a wearable sensing platform that monitors motion (acceleration and posture), ambient light, and skin temperature. It is specifically designed to operate over long periods of time (from days to weeks), and to memorise the user's data locally so that it can be uploaded at a later stage and be analysed by a physician (or in general, a domain expert).

It is currently used in three healthcare-related projects: A decisive project from the early design stages of the Porcupine project that involved the analysis of activity levels of bipolar patients over days. Psychiatrists used and use this type of long-term actigraphy to detect changes in the mood of the patient and predict phases of depression or manic behaviour. Actigraphs are usually worn like a wrist watch by the patients.

A similar project focuses on monitoring activities of elderly users, to automatically asses their independence and detect whether they are still fully able to perform tasks (e.g. household activities). A third healthcare-related application focuses solely on sleep patterns and detection of sleep phases during the user's sleep. This is again based on the observation that certain sleep patterns have different activities and activity intensities associated with them.

In a philosophy of participatory design [2], current users, activity researchers and stakeholders from the previous projects, identified areas where improvements were desirable in terms of usability. One problem is the scalability of deploying the Porcupines in a large trial involving dozens of patients: The feedback from psychiatrists in 


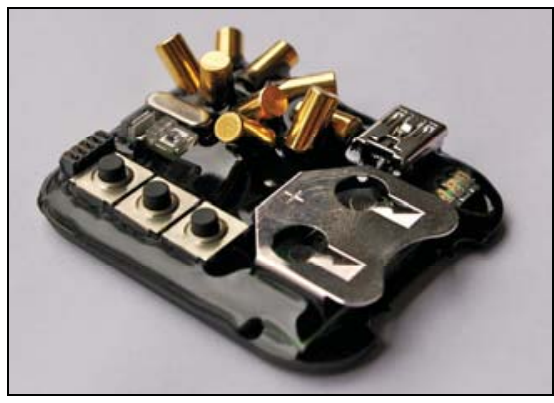

Fig. 1. The current version of the Porcupine prototype

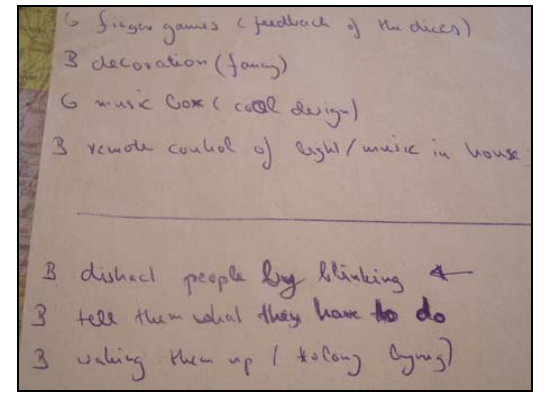

Fig. 2. Subject A ideas; Good and bad ideas signed by $G$ and $B$, respectively

this matter resulted in the replacement of the memory chips by a memory card slot: patients would just replace the card and send it over, while the worn Porcupine would keep on logging. A second problem was the need to change the battery: this would include stocking up on disposable batteries and losing time while changing them. The subsequent Porcupine version used a battery that is rechargeable via the existing USB connector. Experiments after the first versions similarly resulted in buttons and LEDs being cut down in the next version as well.

We analysed the Porcupine performance under five possible usability metrics:

Adaptability - The applications in which the Porcupine sensor unit is used require minimum adaptability. They are worn continuously by the same person, at the same place on the body. Given these, it can be used in almost any context or by any user.

Comfort of use -The Porcupine is worn continuously by users, in a location on the body that is subject to motion. A common place so far was the user's dominant wrist, which tends to be indicative of actions taken. The disadvantage of using this location, however, is that it is in plain view, and that its weight and size are critical.

Reliability - The data is only valid under the assumption that the device is not taken off, unless its data needs to be uploaded, and is thus linked with comfort.

Robustness -The electronics are coated by epoxy, therefore robust to impacts or drops. However, the wrist location does expose the device to splashes of water, which can be problematic if they end up in the battery or USB connectors.

Ease of use -The Porcupine's use is straightforward: most of the time it needs to be worn without maintaining any user interface. Only when configuring or uploading the data from memory to a host computer via USB, some user interaction is required.

From the above analysis, the fact that the Porcupine is worn on a wrist strap still presents usability issues, so we looked for alternatives, by applying the BadIdeas.

\section{Experiment}

The experiment involved seven post-graduate students from Darmstadt University of Technology; the first author moderated the study as a BadIdeas facilitator. Apart from the BadIdeas facilitator, all participants had very strong engineering backgrounds and were familiar with the sensor. Ages varied from 24 to 32 . 
The experiment occurred in three stages: first, a simple illustrative session with the purpose of exploring new uses for Porcupines inside dice; second and focal part, to identify alternative uses for the wrist-worn Porcupine; and third, a post-hoc analysis of the results obtained with the second. The first two phases were carried out during a meeting of roughly 60 minutes. After explaining the experiment goal and task, the two BadIdeas exercises took place, both following the same organization: brief presentation, individual bad ideas writing, verbal sharing ideas with the group and solving in group of the BadIdeas.

An informal environment and structure was preferred as these affect and favour idea generation [1]. This was present in the friendly atmosphere amongst the group and the way information was conveyed and gathered. No formal printed text was used for the brief, neither for the participants to write their ideas; verbal communication and regular blank sheets of paper were used instead (see Fig. 2).

We intentionally omitted the analytic parts of the technique, typical of the second phase, as our ongoing experience shows that people tend to get inhibited, too attached, or even stuck to the structure of the technique. We wanted participants to place all their effort into the problem understanding and generation of solutions, so we removed any tempting rigid structures. No restrictions were given respecting the number of ideas or levels of badness. There were also no domain restrictions.

\subsection{Part I - Porcupine in a Die}

In the first part of the meeting, we wanted to demonstrate to the participants how the BadIdeas technique works and what kind of results it allows one to achieve. Accordingly, we used a playful example and aimed at finding new uses for an existing 'smart die' which has the Porcupine's components embedded in, as detailed in [9].

Roughly the problem brief was: "Suggest us new contexts of use for the Porcupine die that does not include using it in games" (its most obvious application). After explaining this apparently simple task and goal, students still needed "a kick-start" from the facilitator that needed to provide some silly, bad and, apparently unfeasible, examples (e.g. "what if you could eat them?", or "make them out of water") in order to inspire the participants. As can be seen in Table 1, each participant provided on average 2.88 ideas. But, more good than bad ideas, as they were asked to. This evidence is not only observed by us, but also confirmed by the participants, who when asked to go through their written ideas and sign the good and bad ones (see Fig. 2) realised that in fact they wrote more good than bad ideas, even though they were purposely aiming at bad ones. In total, and after eliminating the repetitions, thirteen ideas were supplied. From these, the majority selected the "to use as ice cubes" as the bad idea to transform into good. Embedding the unit in an ice cube was bad because the melted water would damage the circuits of the sensor. But, once one of the participants said the ice was good to cool down drinks and cocktails, someone else said it would be great if that ice cube could tell us when to stop drinking, in case we were driving. Then, someone suggested the sensor was coated with some special material to allow its integration in an ice cube. And, without notice, seconds after, the students were already discussing marketing measures to promote the smart ice cube. 
Table 1. Gathered ideas: good, bad, average and total per participant

\begin{tabular}{lcccc}
\hline Subject & Good Ideas PI & Bad Ideas PI & Total Ideas PI & Total Ideas PII \\
\hline A & 2 & 2 & 4 & 3 \\
B & 2 & 0 & 2 & 0 \\
C & 2 & 1 & 3 & 4 \\
D & 2 & 0 & 2 & 3 \\
E & 0 & 1 & 1 & 0 \\
F & 1 & 0 & 1 & 1 \\
G & 1 & 2 & 3 & 0 \\
H & 5 & 2 & 7 & 3 \\
\hline & Average: & $\mathbf{2 , 8 8}$ & $\mathbf{1 , 7 5}$ \\
\hline
\end{tabular}

\subsection{Part II - Porcupine Wrist Worn Sensor}

The second part of the experiment had a clear usability problem to be solved in respect to a well-defined and stable sensor. Based on the feedback of its users (from patients to doctors), a yet unsolved usability issue of the Porcupine was related to the fact, that it was worn on a wrist strap, which was not always appreciated by the patients. So alternatives were asked from the participants: "Think of a different way of wearing/using the Porcupine. Exclude its current wrist-worn possibility from your suggestions". On average, the participants had now only 1.75 ideas. The first session turned out positive, as participants were already aware of their persistent tendency to switch into generating good ideas instead of pure bad ideas, as suggested and aimed for; no good ideas were written, but also some of the participants were not able to have any ideas.

\subsection{Part III - Post-Hoc Analysis and Results}

After gathering all the ideas and reorganising them by removing the repeated ones, we rated them from five to one according to their inventive potential; five represented the most challenging and one the less challenging ideas.

\begin{tabular}{lc}
\hline Ideas & Rating \\
\hline Hide them in unexpected places to annoy people (in sofa: frighten/surprise peo- & 5 \\
ple, in door: trigger bucket of water, in phone: phone turns off when picked up) & 5 \\
To use in a wig & 5 \\
Recording and erasing data & 4 \\
Ring to punch & 4 \\
Records for only one second & 4 \\
Running out of power & 3 \\
Ball and chain for ankle & 3 \\
Distract people by blinking & 2 \\
To display cryptographic text & 2 \\
Annoying & 2 \\
Waking people up (too long lying) & 1 \\
Blink randomly & 1 \\
Tell people what to do & 1 \\
\hline
\end{tabular}


Accordingly, the most unexpected, odd and surprising ideas were considered as more challenging and the most boring and obvious as the least challenging. The three most inventive ideas were further analysed. We reorganised the experiment's participants into three groups and redistributed the selected ideas to the groups, ensuring that the idea would not be given for transformation to its creator. An email was sent to all participants of the experiment where each group/student was given one idea to solve: i) A Porcupine that records and erases data; ii) A Porcupine to use in a wig; and iii) A Porcupine to hide in unexpected places (sofa, door, phone) to annoy people. To each of the ideas, the students were asked to answer two questions that somehow summarized the remaining three phases of the BadIdeas:

i) What is/are the property/ies that make it that bad? and ii) Is there a context/object/situation in which that property or one of those properties is not bad?

Finally, they were asked to aim at (re)designing the porcupine in order to integrate that feature, by explaining how (by writing), by making sketches or by developing simulations. They were also advised to keep as many records of things as possible. Six of the participants replied and answered their challenges. From our point-of-view, they succeeded as they were all able to complete the exercise and find new uses for the Porcupine. But, surprisingly, they forgot the healthcare and elderly domain and

Table 2. Results of post-hoc session

\begin{tabular}{|c|c|c|c|}
\hline Idea & Subject & $\begin{array}{l}\text { What are the properties } \\
\text { that make it bad? }\end{array}$ & $\begin{array}{l}\text { Is there a context/object/situation in } \\
\text { which the property(ies) is not bad? }\end{array}$ \\
\hline \multirow{2}{*}{ i) } & A & $\begin{array}{l}\text { Monitoring might invade } \\
\text { privacy, erasing impor- } \\
\text { tant data }\end{array}$ & $\begin{array}{l}\text { Erasing is good for solving privacy issues } \\
\text { (e.g., clearing up criminal cases); Porcupine } \\
\text { can decide what to delete/record }\end{array}$ \\
\hline & $\mathrm{H}$ & $\begin{array}{l}\text { Erasing data that is valu- } \\
\text { able }\end{array}$ & $\begin{array}{l}\text { Erasing data when it is not valuable any- } \\
\text { more is good, the erasing would then hap- } \\
\text { pen after a data abstraction phase }\end{array}$ \\
\hline \multirow{3}{*}{ ii) } & $\mathrm{C}$ & $\begin{array}{l}\text { Big and heavy to use on } \\
\text { the head, uncomfortable }\end{array}$ & $\begin{array}{l}\text { The location is good to detect certain head- } \\
\text { related actions, which is great for e.g., } \\
\text { pilots, drivers, policeman }\end{array}$ \\
\hline & $\mathrm{E}$ & I & I \\
\hline & G & $\begin{array}{l}\text { Wig is an unusual loca- } \\
\text { tion, user acceptance }\end{array}$ & $\begin{array}{l}\text { Wig-worn unit could warn if the wig is } \\
\text { shifting out of place, it might also be good } \\
\text { as a party-gadget }\end{array}$ \\
\hline \multirow{3}{*}{ iii) } & $\mathrm{B}$ & 1 & 1 \\
\hline & D & $\begin{array}{l}\text { Embedding it makes it } \\
\text { hard to use them }\end{array}$ & $\begin{array}{l}\text { Hiding them in objects might be good for } \\
\text { users that are hostile towards the unit, } \\
\text { such as prisoners that need to be monitored }\end{array}$ \\
\hline & F & $\begin{array}{l}\text { To annoy is not a valid } \\
\text { purpose }\end{array}$ & $\begin{array}{l}\text { For security, e.g., hiding and annoying } \\
\text { burglars instead of the user, or espionage: } \\
\text { monitoring people unaware of the unit }\end{array}$ \\
\hline
\end{tabular}

went to completely different areas when exploring and solving their bad ideas. From this, we learned that when using the BadIdeas method, we always need to ensure that we remind the participants to go back to the problem domain area, when turning things around and making them good. In the next sections we report the participants' answers also synthesised in Table 2 . 
Porcupine that records and erases data. As bad features the participants stated that a Porcupine would be problematic for privacy reasons and because it could eliminate important data. When analysed more carefully those properties appeared good, if referring to the elimination of criminal cases or if the Porcupine could decide what to delete. For this, a new algorithm was needed, to allow this evolution. In this case, the bad idea was not related with a different place/object of use, so participants did not provide new contexts of use, but they found a way of saving memory use.

Porcupine to use in a wig. Concerning the use of the Porcupine in a wig, participants indicated that it was big, heavy, uncomfortable and even embarrassing to use. Nonetheless, they affirmed that location would change to an advantage if it was used by some specific type of public, e.g. policemen, drivers or pilots.

Porcupine to hide in unexpected places (sofa, door, phone) to annoy people. Finally, participants envisioned possible uses and advantages in the fact of having Porcupines hiding everywhere. The obvious problems were its lack of purpose and also the difficulty of embedding them in the potential hiding places. Once these were resolved, the hidden Porcupine appeared as positive to monitor people that were not aware or were hostile towards the unit, such as burglars and prisoners or patients.

\section{Conclusion and Future Work}

The experiment was experienced as funny and productive. Although vague, these adjectives transmit a positive opinion about the technique. Participants also showed interest about results in the weeks after the study, and kept on mentioning the bad ideas they had. This makes us believe that participants would use the technique again.

Considering the whole process, the use of constraints was important to force the participants to think out of the box, in the first example to keep them away of the most obvious application, and the second, to keep them away of the traditional wristworn Porcupine. At the end, novel alternatives were achieved.

Besides being not as natural or obvious, it was still possible to communicate and continue the BadIdeas process by email. Although, we probably lost the synergetic energy generated by live group discussions. To answer this and other potential open questions, further experiments are needed and should be implemented.

Finally, as referred in other papers [10] and concerning the domain of this paper, as well as other unexplored or relatively new domains, it is crucial to keep the necessity of designing for purpose present. To these domains, guidelines lists are harder to apply and follow and probably should serve more as questioning and thinking starting points than as rules of thumb. So a good practice for evaluation and (re)design is to keep the purpose of the design present and as the main goal to serve.

One of the most important phases in the (re)design of any new appliance or user interface is the one where its core ideas are generated. This paper argues for a novel technique, named BadIdeas, to generate new design ideas and analyse existing products. The generation of ideas is then followed by a process of understanding and modification of ideas. We have applied this technique in a monitored session for the analysis of a research prototype used for actigraphy in several healthcare-related applications, with a team of students that work on and with the prototype. 
The two purposes we had in mind at the beginning of this study were fulfilled as the students were able to conclude the exercise successfully by finding novel user for the Porcupine dice and to identify design alternatives to the wrist-worn Porcupine. In the study, we found that participants require some adjustment to the generation of bad or silly ideas, rather than the usual goal of coming up with good ideas in a certain direction. Especially helpful to this end was the 'warming up' with a mock-up problem, such as the dice in our study, as well as exemplary bad ideas from the facilitator or moderator. Once this was done, the remainder of the study proved to be intuitive, with the participants effortlessly coming up with bad ideas.

One important aspect that stood out was the need for reminding participants to return back to the original area where a usability issue was targeted - as this was not explicitly done in our study, the resulting ideas were often deviating a lot from the intended application.

Acknowledgements. We are grateful to the students involved in the experiment that happily shared their time and actively participated during and after this paper's study.

\section{References}

1. Csikszentmihalyi, M.: Creativity: Flow and the Psychology of Discovery and Invention. Harper Perennial, New York (1996)

2. Dix, A., Finlay, J., Abowd, G D, Beale, R.: Human Computer Interaction. Prentice-Hall, Englewood Cliffs

3. Dix, A., Ormerod, T., Twidale, M., Sas, C., da Silva, P.G.: Why bad ideas are a good idea. In: Proceedings of HCIEd.2006-1 inventivity, Ireland, pp. 23-24 (March 2006)

4. FOBIS - Nordic Foresight Biomedical Sensors (26/6/2007), http://www.sintef.no/fobis/

5. IEEE/EMBS Technical Committee on Wearable Biomedical Sensors and Systems, http://embs.gsbme.unsw.edu.au/ (accessed on the 26/6/2007)

6. Korhonen, I.: IEEE EMBS Techical Committee on Wearable Biomedical Sensors and Systems. Pervasive Health Technologies. VTT Information Technology http://embs.gsbme.unsw.edu.au/ wbss/docs/rep_ambience05.pdf (accessed on 26/6/2007)

7. Svagård, I.S.: FOBIS. Foresight Biomedical Sensors. WORKSHOP 1. Park Inn Copenhagen Airport SINTEF ICT( 6th October 2005) (accessed on 26/6/2007) http://www.sintef.no/ project/FOBIS/WS1/Background\%20for\%20the\%20foresight\%20FOBIS_Svagard.pdf

8. Van Laerhoven, K., Gellersen, H.-W., Malliaris, Y.: Long-Term Activity Monitoring with a Wearable Sensor Node. In: Proc. of the third International Workshop on Body Sensor Nodes, pp. 171-174. IEEE Press, Los Alamitos (2006)

9. Van Laerhoven, K., Gellersen, H-W.: Fair Dice: A Tilt and Motion-Aware Cube with a Conscience. In: Proc. of IWSAWC 2006, IEEE Press, Lisbon (2006)

10. Silva, P.A., Dix, A.: Usability: Not as we know it! British HCI, September, Lancaster, UK. 2007, vol. II, pp.103-106 ( 2007) 